\title{
Inhibition of genes expression of SARS coronavirus by synthetic small inter- fering RNAs
}

\author{
Yi SHI, De Hua YANG, Jie XIONG, Jie JIA, Bing HUANG, You Xin JIN*
}

State Key Laboratory of Molecular Biology, Institute of Biochemistry and Cell Biology, Shanghai Institutes for Biological Sciences, Chinese Academy of Sciences, Shanghai 200031, China

\begin{abstract}
RNA interference (RNAi) is triggered by the presence of a double-stranded RNA (dsRNA), and results in the silencing of homologous gene expression through the specific degradation of an mRNA containing the same sequence. dsRNAmediated RNAi can be used in a wide variety of eucaryotes to induce the sequence-specific inhibition of gene expression. Synthetic 21-23 nucleotide (nt) small interfering RNA (siRNA) with $2 \mathrm{nt}$ 3' overhangs was recently found to mediate efficient sequence-specific mRNA degradation in mammalian cells. Here, we studied the effects of synthetic siRNA duplexes targeted to SARS coronavirus structural proteins $\mathrm{E}, \mathrm{M}$, and $\mathrm{N}$ in a cell culture system. Among total 26 siRNA duplexes, we obtained 3 siRNA duplexes which could sequence-specifically reduce target genes expression over $80 \%$ at the concentration of $60 \mathrm{nM}$ in Vero E6 cells. The downregulation effect was in correlation with the concentrations of the siRNA duplexes in a range of $0 \sim 60 \mathrm{nM}$. Our results also showed that many inactive siRNA duplexes may be brought to life simply by unpairing the 5' end of the antisense strands. Results suggest that siRNA is capable of inhibiting SARS coronavirus genes expression and thus may be a new therapeutic strategy for treatment of SARS.
\end{abstract}

Keywords: SARS, small interfering RNA, Vero E6 cells, EGFP fusion protein, antiviral therapy.

\section{INTRODUCTION}

Severe acute respiratory syndrome (SARS) is a newly emerging infectious disease. It is usually characterized by fever, dry cough, myalgia, and mild sore throat, which progresses to a typical pneumonia. Over 8000 SARS cases and 770 SARS-related deaths were reported to WHO from over 26 countries around the world (http://www.who. $\mathrm{int} / \mathrm{csr} / \mathrm{sars} /$ country/en). SARS is caused by a newly identified virus within the family Coronaviridae [1-3]. This virus has been designated as the SARS coronavirus (SARS-CoV) [4].

RNAi is a natural process by which double-stranded RNA directs sequence-specific silencing of homologous genes. This process is evolutionarily conserved and is found in a wide range of eukaryotic organisms (see for reviews $[5,6]$ ). Specific inhibition of cellular mRNA by RNAi can be triggered in mammalian cells by the introduction of synthetic 21- to 23-nucleotide duplexes of RNA

\footnotetext{
*Correspondence: You Xin JIN

Tel: +86-21-54921222; Fax: +86-21-54921011;

E-mail: yxjin@sibs.ac.cn
}

(siRNA) [7, 8]. This discovery prompted the use of RNAi for specifically inhibiting gene expression and replication of infectious viruses. The replication of a growing number of human pathogenic viruses in cell culture was shown to be inhibited by RNAi, including poliovirus, HIV-1, HCV replicons, hepatitis B virus and influenza virus [9-18]. The successful use of siRNA in mammalian cells encouraged us to apply RNAi to the exploration of anti-SARS strategies.

SARS-Cov is an enveloped, single-stranded, plus-sense RNA virus with a genome of about $30 \mathrm{~kb}[19,20]$. The SARS genome encodes 23 putative proteins which are typical of coronavirus such as 5'-replicase (rep), spike (S), envelope (E), membrane (M), and nucleocapsid (N)-3'. The structural proteins S, E, M and $\mathrm{N}$ are common to all known coronavirus, Playing a role in host cell entry and viron morphogenesis and release [21,22]. During viron assembly, $\mathrm{N}$ binds to a defined packaging signal on viral RNA to form the helical nucleocapsid. $\mathrm{M}$ is localized at intracellular membrane structures. The interaction between the $\mathrm{M}$ and $\mathrm{E}$ proteins and nucleocapsids results in budding through the membrane. These structural proteins were attractive targets for anti-SARS agent development.

Here, we synthesized 26 siRNAs to target the $E, M$, and 
$N$ genes of SARS-CoV and evaluated their effects on viral genes expression in Vero E6 cells. We conclude that the synthetic siRNAs could effectively and sequence-specifically downregulate SARS-CoV genes expression in a dosedependent manner. We obtained 3 siRNAs which could sequence-specifically reduce target genes expression over $80 \%$ at the concentration of $60 \mathrm{nM}$.

\section{MATERIALS AND METHODS}

\section{Preparation of siRNAs}

Sense and antisense strands of siRNA oligonucleotides were synthesized and annealed at $95{ }^{\circ} \mathrm{C}$ for $1 \mathrm{~min}$, followed by incubating at $37^{\circ} \mathrm{C}$ for $1 \mathrm{~h}$ in annealing buffer, $\mathrm{pH} 7.4$, containing $2 \mathrm{mM} \mathrm{Mg}(\mathrm{AC})_{2}$ [23]. To confirm the specificity of the inhibition, the experiments included negative control siRNA duplexes directed against other genes of SARS-CoV. Such as siRNA targeting $M$ gene used as negative controls for siRNA targeting $E$ gene (Tab. 1).

\section{Vectors expressing SARS-CoV genes}

The $E, M$ and $N$ genes expression vectors ( pCDNA3.1/E, pCDNA3.1/M, and pCDNA3.1/N) were constructed and donated by Dr. Youhua XIE (Institute of Biochemistry and Cell Biology, SIBS, CAS). The $E, M$ and $N$ segments were released and inserted into the $\mathrm{pEGFP}-\mathrm{N} 3 \mathrm{~N}$-terminal 'protein fusion mammalian expression vector' (Clontech) to generate 3 new constructs: $p E G F P / E$, $\mathrm{pEGFP} / \mathrm{M}, \mathrm{pEGFP} / \mathrm{N}$. All inserts were sequenced to ensure the orientation and correct reading frame.

\section{Cell culture and transfection}

The Vero E6 cells (from the cell bank of the Institute of Biochemistry and Cell Biology, SIBS, CAS) were cultured in Dulbecco's modified Eagle's medium (DMEM), high glucose (Gibco), supplemented with $10 \%$ heat inactivated fetal calf serum (Hyclone). Transfections of the siRNA duplexes and the plasmids were carried out in 24-well plates using Lipofectamine 2000 reagent (Invitrogen) in accordance with the manufacturer's instructions. $100 \mathrm{nM}$ of the construct and 30-240 nM of siRNA were transiently transfected into Vero E6 cells. The viability of the cells was tested by removing the cells from the culture plate and counting with a haemocytometer after trypan blue staining. The total number of living and dead cells was then calculated.

\section{RT-PCR analysis}

The total RNA of the cells were extracted using TRIZOL reagent (Invitrogen) and digested by RQ1 DNase (RNase free) (Promega) at $37^{\circ} \mathrm{C}$ for $30 \mathrm{~min}$. The DNase was then heat-inactivated at $65^{\circ} \mathrm{C}$ for $10 \mathrm{~min}$. The sequences of the primers for the detection of $E$ gene expression are: 5'ATGTACTCATTCGTTTCGGAA3' (forward) and 5'TTAGACCAGAAGATCAGGAAC3' (reverse). The primers for $M$ gene are: 5'ATGTTACTACAATTTGCCTATTC3' and 5'ACGCTCCTAATTTGTAATAAGA3'. The primers for $N$ gene are: 5'ATGTCTGATAATGGACCCCAA3' and 5'GCCAGGAGT TGAATTTCTTGA3'. The primers for $\beta$-actin are: 5'GTGCCAC CAGACAGCACTGTGTTG3' and 5'TGGAGAAGAGCTATG AGCTGCCTG3'. Single-tube and one-step RT-PCR was performed with the One-step RNA PCR Kit (TaKaRa). Each $50 \mu 1$ reaction mixture contained $1 \mu \mathrm{g}$ extracted RNA, $1 \times$ buffer, $1 \mathrm{mM}$ each deoxynucleotide triphosphate, $0.4 \mu \mathrm{M}$ primer, $5 \mathrm{mM} \mathrm{MgCl}_{2}, 40 \mathrm{U}$
RNase inhibitor, 5 U AMV reverse transcriptase, and $5 \mathrm{U}$ AMVoptimized Taq. After an initial incubation for $30 \mathrm{~min}$ at $50^{\circ} \mathrm{C}$ followed by denaturation at $94^{\circ} \mathrm{C}$ for $4 \mathrm{~min}, 28$ cycles of amplification were performed by using a thermo profile of $94^{\circ} \mathrm{C}$ for $30 \mathrm{sec}, 58^{\circ} \mathrm{C}$ for $30 \mathrm{sec}, 72^{\circ} \mathrm{C}$ for $30 \mathrm{sec}$. The final cycle included an extension at $72^{\circ} \mathrm{C}$ for $10 \mathrm{~min}$. The amplification products were analyzed by electrophoresis on a $2 \%$ agarose gel and stained with ethidium bromide.

\section{Fluorescence microscopy}

The Vero E6 cells were seeded on coverslips-mounted of 6-well plates $\left(5 \times 10^{5}\right.$ cells/well $)$ and transfected as described above. After $48 \mathrm{~h}$, cells were fixed with freshly made $4 \%$ paraformaldehyde in PBS for $30 \mathrm{~min}$ at room temperature and imaged using an Olympus BX-50 fluorescence microscope with $10 \times$ objective. The fields were randomly chosen by a sweep across the coverslips under phase contrast illumination. Randomly chosen fields from differently treated cultures at the same experiment were photographed using the same time of exposure. The proportion of Vero E6 cells expressing GFP fusion proteins was calculated by counting the number of green fluorescent cells against the total number of cells.

\section{Statistical analyses}

Statistical analyses were performed using the student's $t$-test; $P$ values of less than 0.05 were considered as statistically significant.

\section{RESULTS}

siRNAs targeting the $\mathrm{E}, \mathrm{M}$, and $\mathrm{N}$ genes of SARS-CoV

siRNAs were designed to target the open reading frames of the $E, M$, and $N$ genes of SARS-CoV (Tab. 1). The target sequences were directed to a single-stranded region (Fig. 1A), according to the secondary structure of the SARS-CoV RNA predicted by using RNA structure 3.71. Sequences of the form $\mathrm{N} 21$ (where $\mathrm{N}$ indicates any nucleotide) and with a GC content of less than $70 \%$ were selected from this region [24]. The selected 21-nucleotide RNAs, followed by TT, were synthesized chemically by Institute of Biochemistry and Cell Biology, SIBS, CAS.

\section{Downregulation effect of 26 siRNA oligonucleotides}

First, we evaluated the 26 SARS-directed siRNAs for their abilities to suppress SARS-CoV genes expression. The vectors, pCDNA3.1/E, pCDNA3.1/M, and pCDNA3. $1 / \mathrm{N}$, expressing the structural proteins $\mathrm{E}, \mathrm{M}$, and $\mathrm{N}$, were cotransfected with each of the siRNA duplexes into Vero E6 cells. The expression levels of SARS-CoV genes were determined $48 \mathrm{~h}$ later by RT-PCR. The results show that all siRNA duplexes specifically reduced SARS-CoV genes expression to different extents compared with the control (Fig. 1). No. 5, No. 6, and No. 16 siRNA duplexes could suppress SARS-CoV genes expression over $70 \%$ when used at $30 \mathrm{nM}$ (Fig. 1).

\section{Dose-dependent inhibition of SARS-CoV genes expres- sion in cell culture by siRNAs \\ We conducted a dose response analysis by co-transtive}


Tab. 1 Sequences of siRNA duplexes targeting the exons of E, M, and N proteins of SARS-CoV

\begin{tabular}{|c|c|c|}
\hline Name & Sequence* & Target gene \\
\hline \multirow[t]{2}{*}{ No. 1} & 5'-uuaauaguuaauagcguacuuTT-3' & \\
\hline & 3'-TTaauuaucaauuaucgcaugaa-5' & \\
\hline \multirow[t]{2}{*}{ No. 2} & 5'-uuucgugguauucuugcuaguTT-3, & \\
\hline & 3'-TTaaagcaccauaagaacgauca-5' & \\
\hline \multirow[t]{2}{*}{ No. 3} & 5'-uucgauugugugcguacugcuTT-3' & Small envelope protein (E) \\
\hline & 3'-TTaagcuaacacacgcaugacga-5, & \\
\hline \multirow[t]{2}{*}{ No. 4} & $5^{\prime}$-aacgguuuacgucuacucgcgTT-3' & \\
\hline & 3'-TTuugccaaaugcagaugagcgc-5' & \\
\hline \multirow[t]{2}{*}{ No. 5} & 5 '-ugaaggaguuccugaucuucuTT-3' & \\
\hline & 3'-TTacuuccucaaggacuagaaga-5' & \\
\hline \multirow[t]{2}{*}{ No. 6} & 5'-agcuuaaacaacuccuggaacTT-3' & \\
\hline & 3'-TTucgaauuuguugaggaccuug-5' & \\
\hline \multirow[t]{2}{*}{ No. 7} & 5'-ugcugcugucuacagaauuaaTT-3' & \\
\hline & 3'-TTacgacgacagaugucuuaauu-5' & \\
\hline \multirow[t]{2}{*}{ No. 8} & 5'-uauuguaggcuugauguggcuTT-3, & \\
\hline & 3'-TTauaacauccgaacuacaccga-5' & \\
\hline \multirow{2}{*}{ No. 9} & 5'-uagcuacuucguugcuuccuuTT-3' & \\
\hline & 3'-TTaucgaugaagcaacgaaggaa-5' & \\
\hline \multirow[t]{2}{*}{ No. 10} & 5 '-ccagaccgcucauggaaagugTT-3' & \\
\hline & 3'-TTggucuggcgaguaccuuucac-5' & Membrane protein $(\mathrm{M})$ \\
\hline \multirow[t]{2}{*}{ No. 11} & 5 '-uugcgaauggecggacacucc TT-3, & \\
\hline & 3'-TTaacgcuuaccggccugugagg-5' & \\
\hline \multirow{2}{*}{ No. 12} & 5 '-agagaucacuguggcuacaucTT-3' & \\
\hline & 3'-TTucucuagugacaccgauguag-5' & \\
\hline \multirow[t]{2}{*}{ No. 13} & 5'-cgucgcagcguguaggcacugTT-3' & \\
\hline & $3^{\prime}$-TTgcagcgucgcacauccgugac-5' & \\
\hline \multirow[t]{2}{*}{ No. 14} & 5 '-uugcugcauacaaccgcuacc TT-3' & \\
\hline & 3'-TTaacgacguauguuggcgaugg-5, & \\
\hline \multirow[t]{2}{*}{ No. 15} & 5'-aaacuauaaauuaaauacagaTT-3, & \\
\hline & 3'-TTuuugauauuuaauuuaugucu-5' & \\
\hline \multirow[t]{2}{*}{ No. 16} & 5'-gauaauggaccccaaucaaacTT-3' & \\
\hline & 3'-TTcuauuaccugggguuaguuug-5, & \\
\hline \multirow[t]{2}{*}{ No. 17} & 5'-aaggccaaaacagcgccgaccTT-3' & \\
\hline & 3'-TTuuccgguuuugucgeggcugg-5' & \\
\hline \multirow[t]{2}{*}{ No. 18} & 5'-aauaauacugcgucuugguucTT-3' & \\
\hline & 3'-TTuuauuaugacgcagaaccaag-5' & \\
\hline \multirow[t]{2}{*}{ No. 19} & 5'-aaggaggaacuuagauucccuTT-3' & \\
\hline & 3'-TTuuccuccuugaaucuaaggga-5' & \\
\hline \multirow[t]{2}{*}{ No. 20} & 5 '-aagagcuacccgacgaguucg TT-3, & \\
\hline & 3'-TTuucucgaugggcugcucaagc-5' & \\
\hline \multirow[t]{2}{*}{ No. 21} & 5 '-aacaaagaaggcaucguauggTT-3' & Nucleocapsid protein $(\mathrm{N})$ \\
\hline & 3'-TTuuguuucuuccguagcauacc-5' & \\
\hline No. 22 & $5^{\prime}$-aauacacccaaagaccacauuTT-3' & \\
\hline & 3'-TTuuauguggguuucugguguaa-5' & \\
\hline No. 23 & 5'-aauccuaauaacaaugcugecTT-3, & \\
\hline & 3'-TTuuaggauuauuguuacgacgg-5' & \\
\hline No. 24 & 5'-ucaaggaacaacauugccaaaTT-3' & \\
\hline & 3'-TTaguuccuuguuguaacgguuu-5' & \\
\hline No. 25 & 5'-ugaggcaucuaaaaagccucgTT-3' & \\
\hline & 3'-TTacuccguagauuuuucggagc-5' & \\
\hline No. 26 & 5 '-aacguacugccacaaaacaguTT-3, & \\
\hline & 3'-TTuugcaugacgguguuuuguca-5' & \\
\hline
\end{tabular}

*Uppercase letters indicate deoxyribonucleotides. 

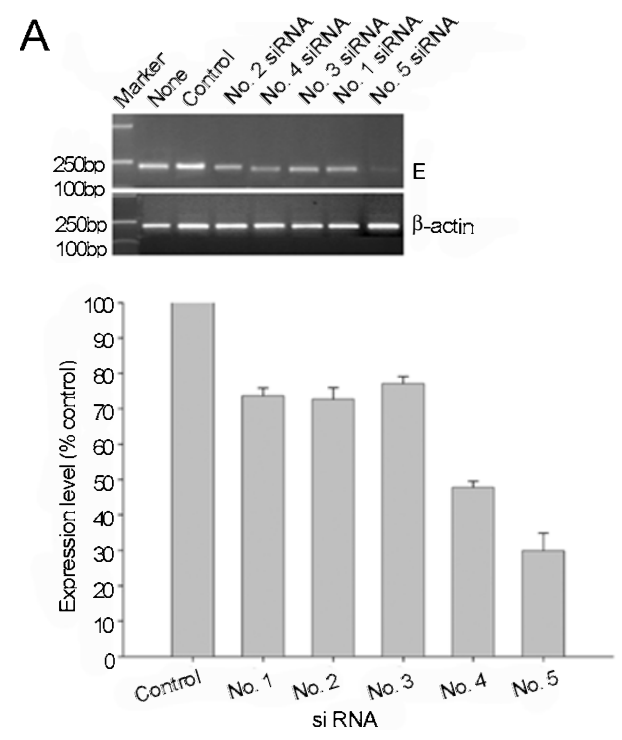

$\mathrm{B}$
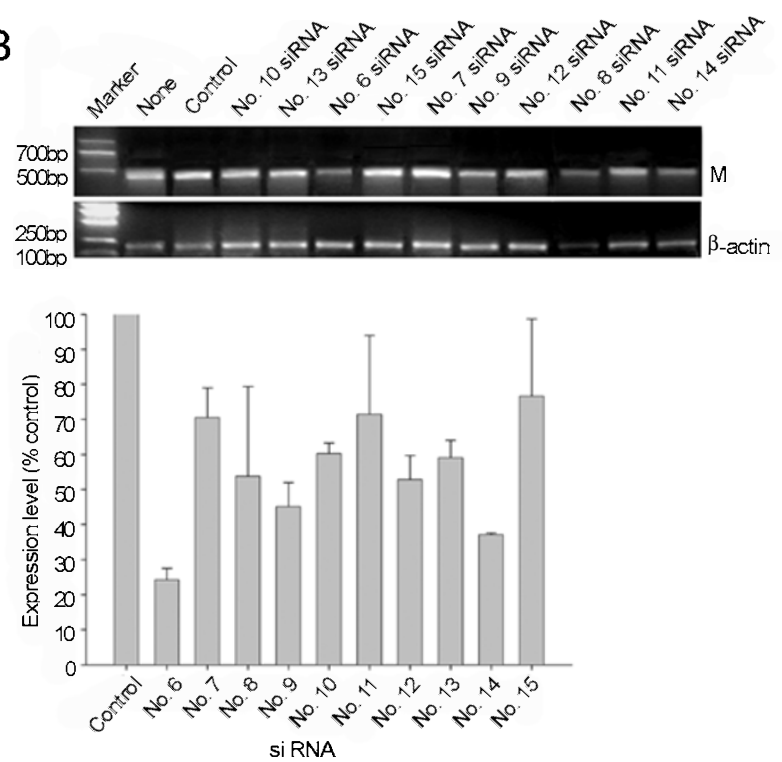

C
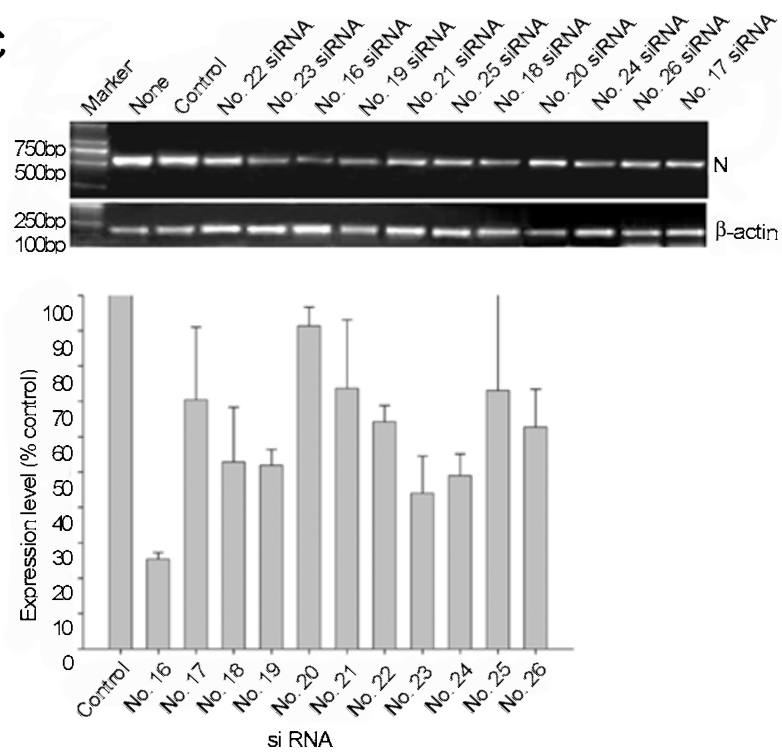
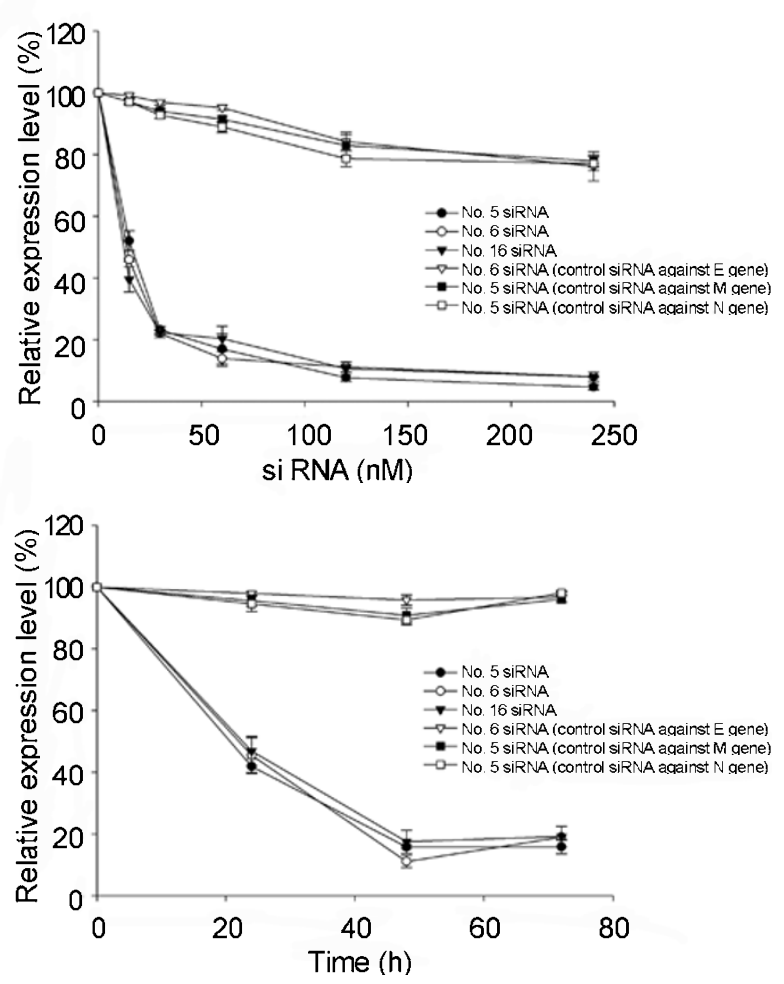

Fig. 2 No. 5, No. 6, and No. 16 siRNA duplexes interfere with expression of viral $E, M$, and $N$ genes. (A) Dose-dependent inhibition of $E, M$, and $N$ genes expression. Vero E6 cells $\left(5 \times 10^{4}\right)$ were cotransfected by $50 \mathrm{ng}$ pCDNA3/E, pCDNA3/M, or pCDNA3/N together with the indicated amounts of the specific siRNA or the irrelevant siRNA as a control and assayed for the expression level of viral $E, M$, and $N$ gene after $48 \mathrm{~h}$ by RT-PCR. (B) Kinetics of inhibition effects of No. 5, No. 6, and No. 16 siRNA duplexes. The expression levels of SARS-CoV $E, M$, and $N$ genes in Vero E6 cells co-transfected with $50 \mathrm{ng}$ pCDNA3/E, pCDNA3/M, or pCDNA3/N together with $60 \mathrm{nM}$ specific siRNA or the irrelevant control siRNA were measured 24,48 , and $72 \mathrm{~h}$ posttransfection. The expression levels of SARS-CoV genes are shown as percentages of the levels secreted by mock-transfected cells (control). The data represent the mean \pm SD of three independent experiments.

$\leftarrow$ Fig. 1 Inhibition of SARS-CoV genes expression by different siRNA duplexes. Vero E6 cells $\left(5 \times 10^{4}\right)$ were transfected with $50 \mathrm{ng}$ plasmid pCDNA3/E, pCDNA3/M, or pCDNA3/N alone (none) or together with $30 \mathrm{nM}$ of each siRNA or with a negative control siRNA (NC), and the expression levels of SARS-CoV genes was measured $48 \mathrm{~h}$ later by RT-PCR. $\beta$-actin served as an internal control. The bar charts below show the results of densitometric analysis shown as percentages of the siRNA negative control. The data represent the mean \pm SD of two independent experiments performed in duplicate. (A) The effects of No. $1 \sim$ No. 5 siRNA duplexes on expression of SARSCoV $E$ gene. (B) The effects of No. $6 \sim$ No. 15 siRNA duplexes on expression of SARS-CoV $M$ gene. (C) The effects of No. $16 \sim$ No. 26 siRNA duplexes on expression of SARS-CoV $N$ gene. 

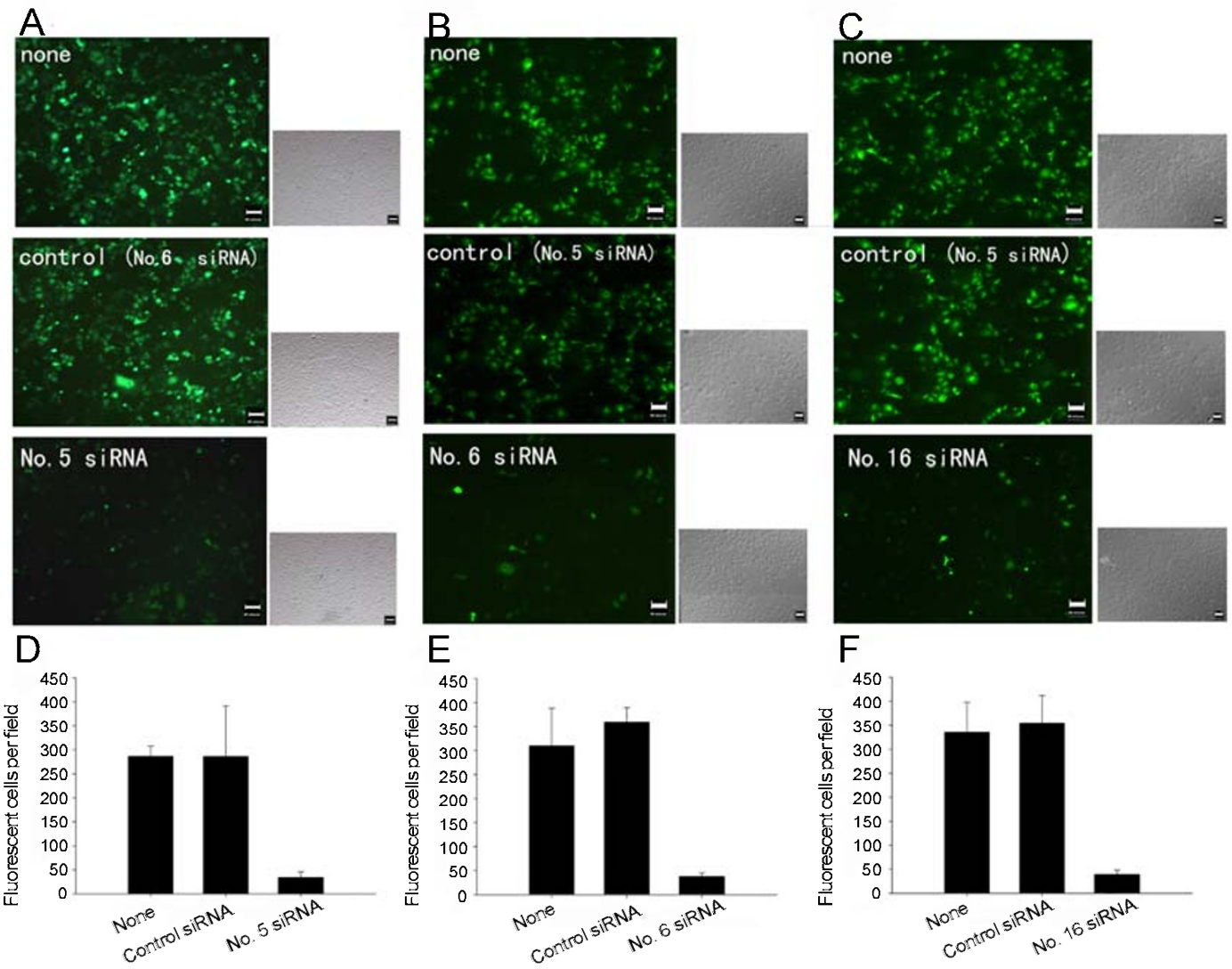

Fig. 3 Effects of No. 5, No. 6, and No. 16 siRNA duplexes on expression of E-EGFP, M-EGFP, and N-EGFP fusion proteins in Vero E6 cells. Fluorescence micrographs of cells (about $1 \times 10^{5}$ ) co-transfected with GFP-fusion protein expression constructs $(100 \mathrm{ng})$ together with irrelevant control siRNA (control), specific siRNA, or without siRNA (none) at the final concentration of $60 \mathrm{nM}$ were taken $48 \mathrm{~h}$ posttransfection. Phase contrast micrographs of the same fields are shown in the right-hand row. (A) Downregulation of expression of E-EGFP fusion protein by No. 5 siRNA. (B) Downregulation of expression of M-EGFP fusion protein by No. 6 siRNA. (C) Downregulation of expression of N-EGFP fusion protein by No. 16 siRNA. (D, E, F) Fluorescent cells per field of view expressing detectable EGFP fusion protein. An average from four fields is shown for each transfection. Data are from a representative experiment. Scale bar: $100 \mu \mathrm{m}$

Tab. 2 Cellular toxicity of the siRNA duplexes

\begin{tabular}{rl}
\hline Type and concentration of siRNA & cells/culture $^{* *}$ \\
\hline None $^{*}$ & $3.68 \times 10^{5}$ \\
$15 \mathrm{nM}$ No.5 siRNA & $3.40 \times 10^{5}$ \\
$30 \mathrm{nM}$ No.5 siRNA & $3.53 \times 10^{5}$ \\
$60 \mathrm{nM}$ No.5 siRNA & $3.31 \times 10^{5}$ \\
$120 \mathrm{nM}$ No.5 siRNA & $3.86 \times 10^{5}$ \\
$240 \mathrm{nM}$ No.5 siRNA & $3.15 \times 10^{5}$ \\
$15 \mathrm{nM}$ No.6 siRNA*** & $3.38 \times 10^{5}$ \\
$30 \mathrm{nM}$ No.6 siRNA & $3.14 \times 10^{5}$ \\
$60 \mathrm{nM}$ No.6 siRNA & $3.55 \times 10^{5}$ \\
$120 \mathrm{nM}$ No.6 siRNA & $3.52 \times 10^{5}$ \\
$240 \mathrm{nM} \mathrm{No.6} \mathrm{siRNA}$ & $3.32 \times 10^{5}$ \\
\hline
\end{tabular}

${ }^{*}$ Transfection was carried out with pCDNA3.1/E alone.

${ }^{* *}$ Number of cells seeded in each well of a 6-well cell culture plate was $2.0 \times 10^{5}$. Viability staining showed not more than $10 \%$ dead cells in all cultures at the end of the experiment $(48 \mathrm{~h})$.

${ }^{* * *}$ Here No. 6 siRNA served as a negative control for $E$ gene. fection Vero E6 cells with increasing amounts of No. 5, No. 6, No. 16 siRNA or negative control siRNA together with pCDNA3.1/E, pCDNA3.1/M, or pCDNA3.1/N. SARS-CoV genes expression levels $48 \mathrm{~h}$ post-transfection were measured. Here the No. 6 siRNA targeting $M$ gene was used as a negative control for detection of $E$ gene expression, whereas the No. 5 siRNA targeting $E$ gene was used as a negative control for that of both $M$ and $N$ genes. The No. 5, No. 6 and No. 16 siRNA duplexes dose-dependently inhibited target genes expression in the range $0 \sim 60 \mathrm{nM}$ (Fig. 2A). At higher doses of siRNA, we observed a slight nonspecific inhibitory effect of negative control siRNA duplexes. We also tested for different toxicities of the siRNA duplex in a set up using $15 \mathrm{nM}, 30$ $\mathrm{nM}, 60 \mathrm{nM}, 120 \mathrm{nM}$, and $240 \mathrm{nM}$ No. 5 siRNA. The total number of living and dead cells revealed no significant 
differences between the No. 5 and the negative control siRNA (No. 6 siRNA) treated cultures (Tab. 2), indicating that the observed downregulation of SARS-CoV genesexpression is not due to toxic effects of these duplexes.

Kinetic study results (Fig. 2B) revealed a continuous increase in the specific inhibition of SARS-CoV genes expression by No. 5 , No. 6 , and No. 16 siRNA from 24 to
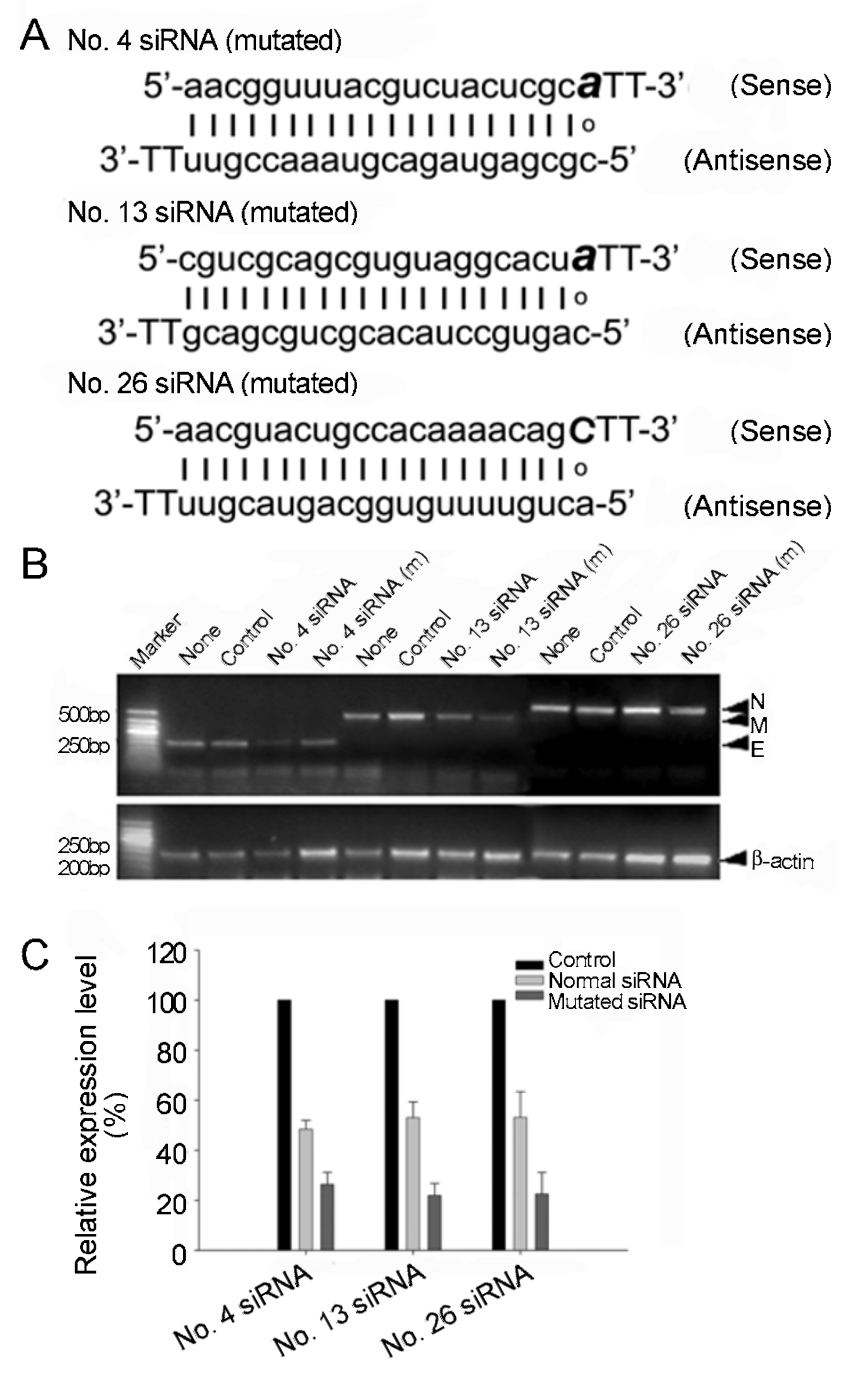

Fig. 4 Effects of mutated siRNA duplexes with a mismatch at the 5' end of antisense strands. (A) The sequences of mutated No. 4, No. 13 , and No. 26 siRNA duplexes. The bold italic letters indicate the mutated nucleotides. Uppercase letters indicate deoxyribonucleotides. (B) Vero E6 cells $\left(5 \times 10^{4}\right)$ were cotransfected by $50 \mathrm{ng}$ pCDNA3/E, pCDNA3/M, or pCDNA3/N together with $60 \mathrm{nM}$ of the mutated siRNA [siRNA (m)] or the irrelevant siRNA as a control and assayed for the expression level of viral E, M, and $\mathrm{N}$ gene after $48 \mathrm{~h}$ by RT-PCR. (C) The bar chart shows the results of densitometric analysis shown as percentages of the siRNA negative control. The data represent the mean $\pm \mathrm{SD}$ of three independent experiments.
$72 \mathrm{~h}$ after transfection. Inhibition of SARS-CoV genes expression reached maximal at $48 \mathrm{~h}$ and started to decline at $72 \mathrm{~h}$. However the effect of siRNA is known to be transient and could last 3-4 days [5].

\section{Effect of siRNA duplexes on the expression of EGFP fused SARS-CoV proteins}

To confirm the effect of No. 5, No. 6, and No. 16 siRNA duplexes on SARS-CoV genes expression, we constructed vectors containing the exon of $E, M$, or $N$ cloned in frame with the EGFP reporter gene and cotransfected Vero E6 cells with these vectors together with the siRNA duplexes. The cells were examined $48 \mathrm{~h}$ post-transfection with fluorescence microscopy. Microscopic examination of the green fluorescence revealed a distinctly reduced expression of the fusion protein in the cells treated with the No. 5, No. 6, and No. 16 siRNA duplexes, compared with cells treated with the negative control siRNA duplexes (Fig. 3).

\section{Effect of siRNA duplexes designed to place the 5 ' end of the antisense siRNA strand in a mismatch}

Recent studies suggested that the thermodynamic properties of siRNA play a critical role in determining the molecule's function $[25,26]$. Functional siRNA duplexes display a lower internal stability at the 5 '-antisense terminal base pair end than nonfunctional duplexes. According to this rule, the nucleotides at position 21 of the sense strands of No. 4, No. 13, and No. 26 siRNA duplexes, which exhibited low inhibition activity, were changed to unpair the 5' end of the antisense strands (Fig. 4A). These mutated siRNA duplexes were transfected into Vero E6 cells together with the expression vectors of the target genes and the expression levels were measured $48 \mathrm{~h}$ posttransfection by RT-PCR. The results showed that the mutated siRNA could reduce target genes expression more effectively (Fig. 4B, 4C). Many inactive siRNA duplexes may be brought to life simply by modifying the sense strands of the siRNA duplexes.

\section{DISCUSSION}

Gene silencing mediated by double-stranded RNA (dsRNA) is a sequence-specific, highly conserved mechanism in eukaryotes. In plants, it serves as an antiviral defence mechanism [27-29]. Recently, the mammalian RNAi machinery has been successfully programmed with siRNAs targeting viral sequences to induce an effective antiviral response. This prompted us to evaluate the effect of siRNA on SARS-CoV. Viruses, especially RNA viruses, are variable due to high mutation rates during replication. The mutated viral proteins can help the viruses to escape immune-system defence mechanisms [30]. These mutations 
may also escape attack by siRNAs. However, the emergence of escape variants resistant to siRNA can be minimized by using siRNA directed against multiple conserved RNA target sequences. The genome of SARS-CoV encoded 23 putative proteins, including four major structural proteins; nucleocapsid (N), spike (S), membrane (M), and small envelope (E) which played essential roles in host cell entry, viral morphogenesis and release [21, 22]. These structural proteins were attractive targets for anti-SARS agent development. Among these proteins, we selected E, $\mathrm{M}$, and $\mathrm{N}$ as the targets of RNAi. The spike protein, a glycoprotein projection on the viral surface, was crucial for viral attachment and entry into the host cell. In addition, variations of S protein among strains of coronavirus are responsible for host range and tissue tropism [31]. The results of genetic sequencing of samples from the latest patient with SARS show variation of $S$ gene in this SARS$\mathrm{CoV}$ strain [32]. Since the S1 subunit of the spike protein is the major antigenic moiety for coronaviruses and is not an essential structural protein, it is prone to have high mutation rates as the virus evolves in host populations [33]. Therefore we don't chose S protein as a target. In this work, we have evaluated the down-regulation effects of 26 siRNA duplexes targeting different sites along the open reading frames of $\mathrm{E}, \mathrm{M}$, and $\mathrm{N}$ proteins. As shown in Fig. 1 , three siRNA duplexes exhibit over $70 \%$ inhibition efficiency, 11 siRNA duplexes showed $40 \sim 70 \%$ inhibition efficiency, and 12 siRNA duplexes reduced target genes less than $40 \%$ at the concentration of $30 \mathrm{nM}$. By modifying the sense strand of siRNA duplex as shown in Fig. 4, the inhibition efficiency of many siRNA duplexes may be improved. The combined use of siRNA duplexes targeting different regions of the virus may increase the efficiency of the treatment and, in turn, will prevent the appearance of viral revertants resistant to the treatment.

Human coronaviruses are usually difficult to culture in vitro, whereas most animal coronaviruses and SARS-CoV can be easily cultured in African Green Monkey Kidney (Vero E6) cells [1]. Direct cytopathic effects of SARS$\mathrm{CoV}$ could be demonstrated on inoculating the viral isolates into Vero E6 cells [3, 19, 34], which make Vero E6 cells a suitable model for the study of the effect of siRNA duplex. Because SARS is dangerous for its high morbidity and mortality rates, it is more safe and convenient to study anti-SARS agent by using Vero E6 cells transfected with SARS-CoV genes expression vectors. In this cell model, we obtained sequence-specific downregulation of SARS$\mathrm{CoV}$ genes expression by synthetic siRNA as seen from the results of RT-PCR (Fig. 1) and from a reduced fluorescence intensity of EGFP to which the SARS-CoV protein $\mathrm{E}, \mathrm{M}$, or $\mathrm{N}$ was fused (Fig. 3). Inhibition was dose dependent in the range of $0 \sim 60 \mathrm{nM}$ and was sustained for
$3 \mathrm{~d}$ after administration of the siRNA (Fig. 2). In addition, this model system can be exploited to further study the pathological functions of SARS-CoV genes.

At present, several potential SARS therapies are under development, such as vaccines $[35,36]$ and interferons $[37,38]$. The use of RNAi in the therapy of SARS would be advantageous in its specificity for the target gene with minimized side effects. However, the interference of SARS-CoV genes expression induced by the use of siRNA duplexes is transient. It will be essential to prolong the expression of siRNA and to ensure its delivery. Current methods for prolonging siRNA expression include the use of plasmids that express endogenously the siRNAs. An alternative approach is to express the siRNA from an integrating viral vector to achieve continuous and prolonged expression. A recent article [39] published during the preparation of our work for report on the anti-SARS activity of a short hairpin RNA (shRNA) targeting the $S$ gene expressed from a U6 promoter suggest that RNAi has potential to inhibit the replication of SARS-CoV from the infected Vero E6 cells. In some cases there may be an advantage to using siRNA because of safety issues regarding the administration of foreign DNA into a patient.

In conclusion, we show that siRNA could be designed to induce an antiviral effect on SARS-CoV genes expression in cells. This approach might provide an effective therapeutic strategy for SARS-CoV infection.

\section{ACKNOWLEDGEMENTS}

We thank Professor Xirui GE for giving us the Vero E6 cell line and Dr. Youhua XIE for donating us three plasmids: pCDNA3/E, pCDNA3/M, and pCDNA3/N. This work was supported by the Grant No. 2003AA208215 from the National High Technology Programs of China and the Grant No. 30270311 from the National Natural Science Foundation of China.

Received, Sep 20, 2004

Revised, Feb 1, 2005

Accepted, Feb 4, 2005

\section{REFERENCES}

1 Drosten C, Gunther S, Doerr HW, et al. Identification of a novel coronavirus in patients with severe acute respiratory syndrome. N Engl J Med 2003; 348:1967-76.

2 Ksiazek TG, Erdman D, Anderson LJ, et al. A novel coronavirus associated with severe acute respiratory syndrome. N Engl J Med 2003; 348:1953-66.

3 Peiris JS, Lai ST, Yuen KY, et al. Coronavirus as a possible cause of severe acute respiratory syndrome. Lancet 2003; 361:131925.

4 Dick T. Update 31: Coronavirus never before seen in humans is the cause of SARS. April 16, 2003. World Health Organization. 
Available: http://www.who.int/csr/sarsarchive/2003 04 16/en/ Accessed May 2, 2003.

5 McManus MT, Sharp PA. Gene silencing in mammals by small interfering RNAs. Nat Rev Genet 2002; 3:737-47.

6 Hammond SM, Caudy AA, Hannon GJ. Post-transcriptional gene silencing by double-stranded RNA. Nat Rev Genet 2001; 2:110-9.

7 Elbashir SM, Lendeckel W, Tuschl T. RNA interference is mediated by 21- and 22-nucleotide RNAs. Genes Dev 2001; 15:188200.

8 Zamore PD, Tuschl T, Sharp PA, Bartel DP. RNAi: doublestranded RNA directs the ATP-dependent cleavage of mRNA at 21 to 23 nucleotide intervals. Cell 2000; 101:25-33.

9 Gitlin L, Karelsky S, Andino R. Short interfering RNA confers intracellular antiviral immunity in human cells. Nature 2002; 418:430-4.

10 Wilson JA, Jayasena S, Richardson CD, et al. RNA interference blocks gene expression and RNA synthesis from hepatitis $\mathrm{C}$ replicons propagated in human liver cells. Proc Natl Acad Sci U S A 2003; 100:2783-8.

11 Randall G, Grakoui A, Rice CM. Clearance of replicating hepatitis $\mathrm{C}$ virus replicon RNAs in cell culture by small interfering RNAs. Proc Natl Acad Sci U S A 2003; 100:235-40.

12 Kapadia SB, Brideau-Andersen A, Chisari FV. Interference of hepatitis $\mathrm{C}$ virus RNA replication by short interfering RNAs. Proc Natl Acad Sci U S A 2003; 100:2014-8.

$13 \mathrm{Ge}$ Q, McManus MT, Chen J, et al. RNA interference of influenza virus production by directly targeting mRNA for degradation and indirectly inhibiting all viral RNA transcription. Proc Natl Acad Sci U S A 2003; 100:2718-23.

14 Coburn GA, Cullen BR. Potent and specific inhibition of human immunodeficiency virus type 1 replication by RNA interference. J Virol 2002; 76:9225-31.

15 Capodici J, Kariko K, Weissman D. Inhibition of HIV-1 infection by small interfering RNA-mediated RNA interference. J Immunol 2002; 169:5196-201.

16 Surabhi RM, Gaynor RB. RNA interference directed against viral and cellular targets inhibits human immunodeficiency virus type 1 replication. J Virol 2002; 76:12963-73.

17 Shlomai A, Shaul Y. Inhibition of hepatitis B virus expression and replication by RNA interference. Hepatology 2003; 37:764-70.

18 McCaffrey AP, Nakai H, Kay MA, et al. Inhibition of hepatitis B virus in mice by RNA interference. Nat Biotechnol 2003; 21: 639-44.

19 Rota PA, Oberste MS, Bellini WJ, et al. Characterization of a novel coronavirus associated with severe acute respiratory syndrome. Science 2003; 300:1394-9.

20 Marra MA, Jones SJ, Roper RL, et al. The genome sequence of the SARS- associated coronavirus. Science 2003; 300:1399-404.

21 Siddell SG. The Coronaviridae. New York: Plenum, 1995.

22 Fields BN, Knipe DM, Howley PM, et al. Fields Virology.
Philadelphia: Lippincott Williams \& Wilkins, 2001.

23 Elbashir SM, Harborth J, Weber K, Tuschl T. Analysis of gene function in somatic mammalian cells using small interfering RNAs. Methods 2002; 26:199-213.

24 Elbashir SM, Martinez J, Patkaniowska A, Lendeckel W, Tuschl T. Functional anatomy of siRNAs for mediating efficient RNAi in Drosophila melanogaster embryo lysate. EMBO J 2001; 20: 6877-88.

25 Schwarz DS, Hutvágner G, Du T, et al. Asymmetry in the assembly of the RNAi enzyme complex. Cell 2003; 115:199-208.

26 Khvorova A, Reynolds A, Jayasena SD. Functional siRNAs and miRNAs exhibit strand bias. Cell 2003; 115:209-16.

27 Vance V, Vaucheret H. RNA silencing in plants - defense and counterdefense. Science 2001; 292:2277-80.

28 Lindbo JA, Silva-Rosales L, Proebsting WM, Dougherty WG. Induction of a highly specific antiviral state in transgenic plants: implications for regulation of gene expression and virus resistance. Plant Cell 1993; 5:1749-59.

29 Voinnet O, Pinto YM, Baulcombe DC. Suppression of gene silencing: a general strategy used by diverse DNA and RNA viruses of plants. Proc Natl Acad Sci U S A 1999; 96:14147-52.

30 Carmichael GG. Medicine: silencing viruses with RNA. Nature 2002; 418:379-80.

31 Kuo L, Godeke GJ, Raamsman MJ, Masters PS, Rottier PJ. Retargeting of coronavirus by substitution of the spike glycoprotein ectodomain: crossing the host cell species barrier. J Virol 2000; 74:1393-406.

32 Parry J. WHO confirms SARS in Chinese journalist. BMJ 2004; 328:65.

33 Ruan YJ, Wei CL, Liu ET, et al. Comparative full-length genome sequence analysis of 14 SARS coronavirus isolates and common mutations associated with putative origins of infection. Lancet 2003; 361: 1779-85.

$34 \mathrm{Ng} \mathrm{ML}$, Tan SH, See EE, Ooi EE, Ling AE. Proliferative growth of SARS coronavirus in Vero E6 cells. J Gen Virol 2003; 84: 3291-303.

35 Gao W, Tamin A, Gambotto A, et al. Effects of a SARS-associated coronavirus vaccine in monkeys. Lancet $2003 ; \mathbf{3 6 2}$ : 1895 6.

36 Sui J, Li W, Marasco WA, et al. Potent neutralization of severe acute respiratory syndrome (SARS) coronavirus by a human $\mathrm{mAb}$ to $\mathrm{S} 1$ protein that blocks receptor association. Proc Natl Acad Sci U S A 2004; 101:2536-41.

37 Moriguchi H, Sato C. Treatment of SARS with human interferons. Lancet 2003; 362:1159.

38 Haagmans BL, Kuiken T, Osterhaus AD, et al. Pegylated interferon-alpha protects type 1 pneumocytes against SARS coronavirus infection in macaques. Nat Med 2004; 10:290-3.

39 Zhang Y, Li T, Chang Z, et al. Silencing SARS-CoV Spike protein expression in cultured cells by RNA interference. FEBS Lett 2004; 560:141-6. 